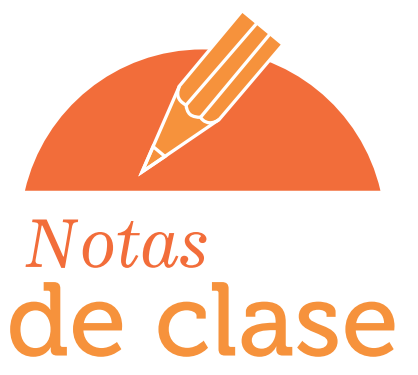

Apropiación social del conocimiento

Generación de contenidos impresos https://repository.ucc.edu.co/handle/20.500.12494/7375

\title{
ESTRATEGIAS DE APRENDIZAJE PARA HISTORIAY EPISTEMOLOGÍA DEL CUIDADO DE ENFERMERÍA
}




\section{ACERCA DE LA AUTORA}

Elveny Laguado Jaimes, Magíster en Enfermería. Profesora asistente del programa de Enfermería, sede Bucaramanga, Colombia.

Correo electrónico:

elveny.laguado@campusucc.edu.co

CvLAC: https://scienti.minciencias.gov.co/cvlac/

visualizador/generarCurriculoCv.do? cod_

$r h=0000722235$

Orcid: https://orcid.org/0000-0001-7169-6912

Google Scholar: https://scholar.google.com/

citations? user=j4IrfiYAAAAJ\&hl=th

\section{Cómo CITAR ESTE DOCUMENTO}

Laguado Jaimes E. Estrategias de aprendizaje para historia y epistemología del cuidado de enfermería [Generación de contenidos impresos N. $\left.{ }^{\circ} 3\right]$. Universidad Cooperativa de Colombia; 2020. https://doi.org/10.16925/gcnc.16

\section{NOTA LEGAL}

El presente documento de trabajo ha sido incluido dentro de nuestro repositorio institucional como Apropiación social de conocimiento por solicitud del autor, con fines informativos, educativos o académicos. Asimismo, los argumentos, datos y análisis incluidos en el texto son responsabilidad absoluta del autor y no representan la opinión del Fondo Editorial o de la Universidad.

\section{DISCLAIMER}

This coursework paper has been uploaded to our institutional repository as Social Appropriation of Knowledge due to the request of the author. This document should be used for informational, educational or academic purposes only. Arguments, data and analysis included in this document represent authors' opinion not the Press or the University.

(c) (1) () $\Theta$ Este documento puede ser consultado, descargado o reproducido desde nuestro repositorio institucional (http://repository.ucc.edu.co/handle/20.500.12494/7369) para uso de sus contenidos, bajo la licencia de Creative Commons Reconocimiento-NoComercial-SinObraDerivada 4.0 Internacional. http://creativecommons.org/licenses/by-nc-nd/4.0/ 


\section{TABLA DE CONTENIDO}

INTRODUCCIÓN

Referencias 6

$\begin{array}{ll}\text { Propósito del curso y competencias } & 7\end{array}$

UNidAd I. HeCHOS HISTÓRICOS Y EVOLUCIÓN DEL CUIDAdO 7

Prefacio 7

Tema: aspectos generales de la evolución del cuidado

Desarrollo 9

Ejercicio 9

Referencias 10

$\begin{array}{ll}\text { Bibliografía complementaria } & 10\end{array}$

Unidad II. PATRONES de CONOCIMIENTO EN ENFERMERÍA 11

Prefacio 11

$\begin{array}{ll}\text { Tema } & 11\end{array}$

Desarrollo 12

$\begin{array}{ll}\text { Ejercicio } & 12\end{array}$

$\begin{array}{ll}\text { Referencias } & 14\end{array}$

Bibliografía complementaria $\quad 14$

Apartado final 15 


\title{
01 ESTRATEGIAS DE APRENDIZAJE PARA HISTORIA Y EPISTEMOLOGÍA DEL CUIDADO DE ENFERMERÍA
}

\author{
Elveny Laguado Jaimes
}

\section{Resumen}

Gracias a la nota de clase, los alumnos y profesores de Enfermería disponen de un material que les permitirá desarrollar estrategias para apropiarse de los aspectos relacionados con el origen de la enfermería y su influencia en el avance del cuidado como esencia del quehacer profesional. Los hechos históricos desde una mirada crítica y reflexiva permiten valorar y reconocer en las diferentes épocas de la historia situaciones del contexto social, político y económico que contribuyeron a los cambios para el presente de la profesión y los desafíos del futuro; esto debido a que la historia, como parte inherente de una sociedad, se escribe de manera permanente por los modos cambiantes de la humanidad. Hay un presente con legados históricos que fortalecen una profesión que está evolucionando constantemente en cuanto a construcción como ciencia, con los referentes teóricos que los alumnos identifican por primera vez. Asimismo, la forma como se comprende, valora e interpreta contribuirá a que se empoderen y lideren cambios en su práctica profesional y se fortalezca la calidad del cuidado y la identidad profesional.

Palabras claves: historia de la enfermería, evolución del cuidado, epistemología, estrategias de aprendizaje, patrones de conocimiento. 


\section{INTRODUCCIÓN}

En la Educación en Enfermería, el abordaje de la historia del cuidado de enfermería es un desafío, puesto que la reconstrucción del pasado para el alumno requiere análisis, reflexión e interpretación de los hechos que enmarcaron el desarrollo de la enfermería como profesión, mediante una historia crítica sin mitos o estereotipos. La enseñanza de la historia en enfermería implica una constante indagación sobre los desafíos que plantea el ser enfermeros [1].

Considerar el valor de los cambios en la historia permite comprender las prácticas actuales en enfermería, y la continuidad de las modificaciones de la estructura social, política y estatal es en sí historicidad para el futuro. Por lo tanto, es de vital importancia revisar los múltiples contextos del origen histórico, no solo el análisis de la información y las diversas fuentes, sino también el reconocimiento del contexto histórico, temporal, cultural, social y político [2, 3].

En relación con el avance del cuidado en enfermería y su profesionalización, en diferentes fuentes históricas se menciona que el cuidado doméstico se caracteriza por acciones de supervivencia y que está dado exclusivamente a la mujer. Posteriormente, bajo la influencia religiosa, se presenta el cuidado vocacional y la institucionalización del cuidado con la creación de espacios para la atención de los enfermeros; ahora, con los avances, el cuidado se transforma con la inclusión de la enfermería moderna hacia la profesionalización del cuidado [4].

Como parte de la profesionalización, el auge de los referentes teóricos con Florence Nightingale y su teoría naturalista establece la necesidad de avanzar en la ciencia del cuidado que fundamente el quehacer profesional. Por esto, la enseñanza de las expresiones del conocimiento, denominadas patrones del conocimiento, permite que el alumno de enfermería inicie un proceso de apropiación del marco epistemológico.

Es importante considerar que en el proceso educativo los preconceptos del alumno son parte importante ante los nuevos conocimientos y las conexiones que pueda realizar, para generar un aprendizaje significativo. Incluir el análisis crítico y reflexivo en las actividades permite al estudiante identificar y resolver problemáticas acordes a las necesidades actuales y asumir durante su formación un compromiso con su desarrollo profesional permanente, que responda a los retos de la sociedad. Otro factor importante es la motivación presente en la cotidianidad, que da sentido a los propósitos y acciones. Así, las estrategias de enseñanza aprendizaje, acordes a los aspectos mencionados anteriormente, contribuyen a que el alumno se apropie de la temática de forma participativa. 


\section{REFERENCIAS}

[1] Cerdá JM, Ramacciotti K. Historia y cine documental en la enseñanza de enfermería. CyA [Internet]. 2018 jun. 19 [citado 2020 nov. 19];(26):87-100. Disponible en: https://bibliotecavirtual.unl.edu.ar/publicaciones/ index.php/ClioyAsociados/article/view/7279

[2] Cammarota A, Faccia K. Enseñar Historia en las carreras de salud. Interface (Botucatu) [Internet]. 2018 dec. [citado 2020 nov. 19];22(67): 1003-15. Disponible en: http://www.scielo.br/scielo.php?script=sci_arttext\& pid=S1414-32832018000401003\&lng=en

[3] Cerdá JM, Ramacciotti K. La enseñanza de Historia Social en la carrera de Enfermería. Interface (Botucatu) [Internet]. 2018 dec. [citado 2020 nov. 19];22(67): 993-1002. Disponible en: http://www.scielo.br/scielo. php?script=sci_arttext\&pid=S1414-32832018000400993\&lng=en

[4] Martínez Martin ML. Chamorro Rebollo E. Historia de la Enfermería. Evolución histórica del cuidado enfermero. Elsevier; 2011. p. 176 


\section{PROPÓSITO DEL CURSO Y}

\section{COMPETENCIAS}

Durante el desarrollo del curso de Historia y Epistemología del Cuidado, la lectura de material de diversas fuentes es la base que le permitirá al alumno demostrar su apropiación mediante la competencia establecida: explicar el proceso de evolución del cuidado a través de la historia, mediante evidencias concretas que desarrollará el alumno y que se explicarán en la primera unidad de esta nota de clase.

El curso cuenta con otra unidad que corresponde al marco epistemológico, para el cual el alumno desarrolla la competencia: analizar los conceptos que forman el marco epistemológico de enfermería en la evolución del cuidado a través de la historia. En esta nota de clase los patrones de conocimiento se exponen con estrategias que faciliten el proceso, para que el alumno adquiera la competencia.

\section{UNIDAD I. HECHOS HISTÓRICOS Y EVOLUCIÓN DEL CUIDADO}

\section{Prefacio}

En la educación de enfermería, los diferentes planes de estudio asumen la historia de la enfermería como un curso que en la mayoría de los casos se da en los primeros niveles. En el inicio, para un alumno es un curso teórico que contiene información y material de diversas fuentes que debe revisar y leer. Por lo tanto, el reto del profesor es que el alumno identifique la construcción de hechos históricos que incidieron en el desarrollo de enfermería, pero mediante un análisis crítico y reflexivo. Para esto, el estudiante necesita indagar diversas estrategias que cumplan el propósito para que, al finalizar el curso, comprenda el legado históri$\mathrm{co}$, que forma parte de la identidad profesional y del compromiso por seguir transformando el quehacer profesional.

Para el desarrollo del curso, se consultan fuentes de autores que han escrito libros de historia, pero también artículos de investigación histórica y estudios relacionados con el marco epistemológico del cuidado. Esto le permitirá al alumno contar con material diverso, que orienta las reflexiones y los análisis de la información, con las estrategias de aprendizaje propuestas.

\section{TEMA: ASPECTOS GENERALES DE}

\section{LA EVOLUCIÓN DEL CUIDADO}

En los cambios de la humanidad a través de la historia, el cuidado se configuró en sus inicios con el propósito de subsistir; pensamiento que fue aceptado por la colectividad. El origen de las prácticas de cuidado se relaciona con la conservación del grupo, lo cual es especialmente dada a las mujeres que, en particular, están relacionadas con la fecundidad. Estas actividades de cuidado se daban de forma intuitiva, no existía razonamiento previo.

Luego, ante los cambios sociales y la influencia de los preceptos religiosos, surgió el concepto de ayuda en la práctica del cuidado, y se fortalece con la institucionalización del cuidado, unido al inicio del cristianismo que va impregnando la filosofía del hombre. La caridad, entonces, encuentra su sentido en el concepto de ayuda denominado: vocacional cristiano, caritativo. En la Iglesia oriental proliferan los hospicios denominados "nosocomia", que en Occidente se denominaron "xenodochium", en el siglo vi.

Ante la conceptualización del cuidado como ayuda, Hernández Conesa [1], refiere que esta caridad asociada a la ayuda de los pobres fue una barrera para el avance de la enfermería como profesión. El cuidado de enfermería visto desde una perspectiva caritativa no avanza 
en la constitución de gremio, pues predomina la dimensión espiritual, sin la necesidad de aprendizaje, calidad y salarios.

Ahora bien, el contexto social e histórico de la humanidad conlleva cambios que aportan a la transformación de los cuidados. Así es como durante el proceso de la reforma protestante, el concepto de ayuda se modifica, y de esta manera los cuidados de enfermería pierden su sentido salvador; los hospitales se cierran, expulsan a los monjes y monjas, y no sustituyen a los que cuidan a los enfermos. Este deterioro de los cuidados enfermeros y de las personas que los practican conforma en el mundo protestante la época oscura de la enfermería.

Durante 300 años, no se evidencia ningún cambio a nivel institucional de enfermería. Al surgir la necesidad de crear escuelas para las enfermeras, empieza el cuidado a recobrar el reconocimiento social perdido, con los cual surgen entrenamientos prácticos en labores domésticas y cuidado general del enfermo. Así, la profesionalización se da fuera del concepto caritativo, como una necesidad social creada por la comunidad protestante ante la atención de los enfermos, surgiendo la profesionalización-caritativa-vocacional.

Asimismo, ante los cambios progresistas en lo social y económico del siglo XIX, revolucionan los medios de transporte y la concentración de personas en las ciudades, ambiente propicio para enfermedades y epidemias, siendo una época también de grandes avances científicos. Los primeros programas de instrucción del momento respecto a los cuidados de enfermería en la concepción de Nightingale, se orientan a la prevención de la enfermedad y promoción de la salud, ante el concepto de cuidados curativos.

La concepción de Nightingale [2], supone la consideración de la enfermería como una opción profesional; su método de trabajo involucra lectura, revisión de datos y observación.
Ante la necesidad de avanzar en el cuidado profesional, se asumen, en torno a una conducta moral de la enfermería, tareas técnicas delegadas. Las competencias en enfermería, el arte y la ciencia no son visibles. Luego, mediante el proceso de construcción disciplinar, el reconocimiento jurídico y la necesidad de capacitación profesional fortalecen el reconocimiento de la enfermería como profesión.

Con la implementación de los programas de educación en las escuelas de enfermería en Europa se fundamenta el carácter moderno como profesión; a Nightingale se le atribuye la introducción de la ciencia, higiene y el hecho de atraer a mujeres consideradas morales y sobresalientes en la comunidad. Durante su progresivo avance, la enfermería ha estado asociada a la espiritualidad y la enfermería moderna occidental; las bases de su filosofía de cuidado y preocupación humanitaria están dadas por las influencias de la cristiandad y son denominadas herencia religiosa [3].

Para esta época, cuando se conmemoran los 200 años del natalicio de Florence Nightingale - quién se menciona con frecuencia en la educación en enfermería-, es importante plantear los aspectos que han formado parte de los desarrollos conceptuales en la construcción del conocimiento en enfermería en América Latina. El paso a la universidad fue un avance, pero al mismo tiempo llevó a que se consolidaran los conceptos propios de la disciplina, una visión amplia, crítica, autónoma y acorde a las necesidades que demandan las personas. La exigencia de cuidados con calidad y bienestar, para una calidad de vida posible, contribuye al desarrollo de la disciplina [4].

Cabe destacar que en la historia de la enfermería es constante afrontar dificultades, riesgos que en cada época se superaron, sistemas de salud cambiantes y abordaje de la salud y la enfermedad que marcan pautas para la constante trasformación del colectivo de enfermería. Es por esto que se reconoce la historia 
como experiencias que aportan a la construcción de estrategias que permitan afrontar los desafíos del futuro.

En cuanto a la historia, es importante agregar que se considera la enfermería como una ciencia dinámica y global, la cual da una visión del fenómeno humano; por esto es dinámica, puesto que estudia el cuidado a través del tiempo, en cada época y con cambios social y globales, y permite identificar aquellos factores que influyen en las formas de cuidado en un momento histórico determinado [5].

\section{DESARROLLO}

Es importante retomar el hecho de que, para apropiarse de la historia en enfermería, se requiere un análisis crítico y reflexivo de la información; de esta forma, la interpretación de la información y cómo influye en los cambios y avances del cuidado le permitirá al alumno apreciar y apropiar el tema de trabajado.

Por lo anterior, las estrategias propuestas son:

- Lectura crítica de artículos que relatan la historia de la enfermería, consultados en las bases de datos.

- Desarrollo de un conversatorio grupal, para exponer los análisis de la información consultada.

- Socializan de la información consolidada mediante un video, haciendo énfasis en hechos históricos que influyen en la evolución del cuidado en la historia.

- Portafolio: recopilación de información relacionada con la consulta de referentes históricos. Los estudiantes elaboran relatorías e imágenes consultadas y así confirman una secuencia de reportes organizados por las etapas de la historia- Con este portafolio se fomenta el aprendizaje reflexivo, autónomo y participativo.

\section{EJERCICIO}

1. Mediante aprendizaje colaborativo, cada grupo de estudiantes busca en las bases de datos: Scopus, Sciencie Direct, Sage, Scielo, Redalyc, utilizando las palabras claves: history and nursing; history and care.

Cada grupo debe extraer los hechos históricos y su relación con los avances del cuidado, y organizar en un cuadro según el ejemplo que se muestra en la tabla 1.

Tabla 1

Relación Hechos históricos con evolución del cuidado

\begin{tabular}{|c|c|c|}
\hline $\begin{array}{c}\text { Época de } \\
\text { la historia }\end{array}$ & $\begin{array}{c}\text { Hechos } \\
\text { históricos }\end{array}$ & Influencia en el cuidado \\
\hline Media & Guerras & $\begin{array}{c}\text { Identificar la disciplina, } \\
\text { el uso del uniforme }\end{array}$ \\
\hline Moderna & $\begin{array}{c}\text { Incrementan } \\
\text { las epidemias }\end{array}$ & $\begin{array}{c}\text { Requerimiento de cuidadores } \\
\text { con una preparación adecuado a } \\
\text { las necesidades de las personas. }\end{array}$ \\
\hline
\end{tabular}

\section{Fuente: elaboración propia}

2. Portafolio: recorriendo la historia de enfermería

Por grupos de trabajo colaborativo de máximo tres personas, los alumnos consultan hechos históricos (máximo tres) en cada etapa: antigua, media, moderna, contemporánea y actual. Esto debe estar acompañado de imágenes relacionadas con estos hechos. El documento debe elaborarse en formato Word, Power Point u otro formato compartido en línea. Es importante tener en cuenta las normas de la Asociación Americana de Psicología (APA) para las referencias, escritura organizada, ortografía y uso adecuado del lenguaje. Es importante resaltar que el uso de recursos audiovisuales en el portafolio contribuye a que los alumnos intercambien opiniones, conexiones y discusiones en las articulaciones propuestas por cada uno de los grupos de trabajo. 


\section{REFERENCIAS}

[1] Hernández Conesa J. Historia de la Enfermería: Un análisis histórico de los cuidados de enfermería. Madrid: McGraw-Hill, Interamericana; 1995.

[2] Burgos Moreno M, Paravic Klijn T. Enfermería como profesión. Revista cubana de enfermería. 2009;25(1-2), 0-0.

[3] Velandia Mora AL. Historia de la enfermería en Colombia. Bogotá: Universidad Nacional de Colombia;1995.

[4] García-Carpintero BE. Reflexión del papel de la enfermría a lo largo de la Historia. Enf Global [Internet]. 2007 nov. 1 (citado 19 nov. 2020);6(2). Disponible en: https://revistas.um.es/eglobal/article/view/358

[5] Siles González J. Historia cultural de enfermería: reflexión epistemológica y metodológica. Av Enferm. [Internet]. 2010 feb. 1 (citado 19 nov, 2020);28(E):120-8. Disponible en: https://revistas.unal.edu.co/index. php/avenferm/article/view/21456

\section{BiBLIOGRAFÍA COMPLEMENTARIA}

García-Carpintero Blas E, Siles González J, Martínez Roche ME, Martínez de Miguel E, González Cervantes S, Pulido Mendoza R. The student as protagonists of their learning: the need to use the portfolio in nursing within the context of higher education. Index de Enfermería. [Internet]. 2015 (citado 19 nov. 2020);24(12):93-97. Disponible en: https://dx.doi.org/10.4321/S1132-12962015000100021

Siles González J. Historia de la enfermería: una aportación epistemológica desde la perspectiva cultural de los cuidados. Cultura de los Cuidados. 2008;12(24):5-6. 


\section{UNIDAD II. PATRONES DE CONOCIMIENTO EN ENFERMERÍA}

\section{PREFACIO}

Durante el proceso educativo, los alumnos identifican las expresiones del conocimiento en diferentes estudios realizados por enfermeras en situaciones específicas como parte de procesos investigativos, estudios de casos o narrativas. Esto les permite aproximarse a una realidad expresada por otros, y esa experiencia aporta a la comprensión e identificación de características propias de cada patrón de conocimiento. Por lo anterior, en la revisión de los patrones de conocimiento, el alumno asume un desafío, puesto que con sus características y la manera como se presenta en situaciones de cuidado, valora e integra las intervenciones que desarrolla en sus prácticas y argumenta y fundamenta las acciones de cuidado a partir de los referentes teóricos contribuye a fortalecer las actitudes y aptitudes como líder en su ejercicio profesional.

\section{TEMA}

La integración entre la ciencia y la profesión de enfermería permite que la enfermera, en su quehacer profesional, realice el cuidado en diferentes periodos del ciclo vital y de contextos diversos. La persona se construye con una visión holística y se enfoca en las respuestas humanas ante situaciones de salud o enfermedad que requieren planes de intervención con calidad. Así, ante la impronta de fundamentar el cuidado, han surgido planteamientos filosóficos, valores, estructuras teóricas, comportamientos y patrones de conocimiento que orientan y describen las características y formas de pensar en relación con los fenómenos de interés de la disciplina. Producto de la revisión y trabajo investigativo, Bárbara Carper, en 1978, escribe los patrones del conocimiento como los que le dan soporte a la enfermería. Estos los denomina como: empírico (ciencia de enfermería), estético (arte de enfermería), conocimiento personal y ético, este último como el componente moral [1].

Existen otros patrones que complementan lo anterior, propuestos por White en 1995, por ejemplo, el sociopolítico para identificar los contextos del paciente y la enfermera; estos ambientes de interacción se vuelven esenciales para una comprensión total y para entender la importancia de la enfermería en la sociedad. Por su parte, Jacobs y Chinn, en 1999, agregan el patrón emancipatorio como el análisis y la reflexión crítica de las situaciones, que permite identificar el contexto social y político del cuidado de la salud y la práctica en enfermería [1]. Una parte importante de la educación en enfermería la constituye la epistemología en enfermería, de la cual debe fomentar conciencia de su relevancia para la integración de los patrones de conocimiento [2].

Es importante referir que los patrones del conocimiento están descritos con características particulares que permiten diferenciar un proceso de análisis de situaciones o de reportes de investigación. Por lo tanto, para un patrón de conocimiento empírico son válidos los referentes teóricos propios de la disciplina y de otras; esto permite fundamentar las acciones y respuestas que requieren los sujetos de cuidado a sus necesidades; asimismo, el patrón de conocimiento estético evidenciado por expresiones no verbales, acciones terapéuticas, empatía como principal característica, el patrón de conocimiento personal —el más difícil de enseñar, puesto que es la expresión de sí mismo en un contexto interpersonal-, el ético que es guiado por el código ético, el asumir los principios y valores en las conductas y el ejercicio profesional $[3,4,5]$.

Los patrones de conocimiento sociopolítico y emancipatorio no han tenido un desarrollo teórico; sin embargo, en el primero se identifican 
problemas sociales y políticos del contexto, aspectos que influyen en los entornos físicos, psicológicos y del cuidado de la salud y su relación con la profesión de enfermería, que incluye liderazgo y gestión; en el segundo, la estrategia clave implica la reflexión colectiva de la situación del cuidado y su proceso de transformación ante los problemas que emergen de los cambios constantes del contexto [3].

En la educación de enfermería, se problematiza la teoría y la práctica, en razón a su génesis. Mientras que la práctica se da de manera intuitiva desde el comienzo de la vida, la teoría inicia con las propuestas de la pionera en enfermería, Florence Nightingale, y posteriormente otras autoras desarrollan sus referentes teóricos; sin embargo, las teorías médicas han sido con mayor frecuencia el soporte de la práctica enfermera. En las últimas décadas, ante el interés en la investigación y la utilidad de las teorías, se requiere la reflexión para la apropiación de estas últimas [6].

En la introducción al marco epistemológico que relata el conocimiento de la disciplina de enfermería, el alumno identifica un componente importante que fundamenta la profesión. Otras disciplinas reconocidas como las ciencias biológicas y humanas complementan la comprensión del ser humano, puesto que, ante la premisa de dar un cuidado holístico, el abordaje de la persona es biopsicosocial, espiritual y se encuentra en interacción con el entorno. Así, al apropiarse de argumentos basados en diversas disciplinas, el estudiante responde a las necesidades e interviene las respuestas humanas relacionadas con las situaciones de cuidado [7].

\section{DESARROLLO}

Durante el proceso educativo, los patrones de conocimiento expuestos en el aula sin la experiencia de un encuentro con el sujeto de cuidado pueden ser abstractos, en la medida en que se asimilan sus características y no se validan en un contexto real próximo; por lo tanto, se requieren estrategias que permitan vivenciar una experiencia y verificar los patrones de conocimiento.

Dentro de las estrategias de aprendizaje para realizar, están las siguientes:

- Lectura crítica de estudios relacionados con la temática: patrones de conocimiento en situaciones específicas de cuidado.

- Análisis de artículos de investigación en los que, mediante la revisión de los aspectos que conforman el artículo, se describan las características de cada patrón de conocimiento.

- Discusión de caso: se puede desarrollar con la revisión de una película en la que se pueda evidenciar acciones de enfermería.

- Situaciones de enfermería: análisis de los patrones de conocimiento en una narrativa de enfermería, herramienta que permite la construcción de actos humanos significativos a partir de la interacción enfermera-paciente, entender la vivencia de las personas en su propio contexto y reflejar a la enfermera como un ser humano.

\section{EjERCICIO}

Mediante las actividades que se muestran en la tabla 2, el alumno se apropia de las características de cada patrón de conocimiento; asimismo, puede consultar los referentes expuesto en el texto anterior o hacer búsqueda en bases de datos. Es importante que se desarrollen las actividades y se haga discusión en grupo de los resultados; esto permite intercambio de saberes, aclarar dudas y fomentar la reflexión crítica.

1. En la tabla 2, se expresan las características de los patrones de conocimiento. El 
estudiante debe ver el ejemplo inicial e identificar a cuál corresponde.

Tabla 2

Características patrones de conocimiento

\begin{tabular}{|c|c|}
\hline Características & $\begin{array}{c}\text { Patrones de } \\
\text { conocimiento }\end{array}$ \\
\hline Empatía & Patrón estético \\
\hline Enfermería basada en la evidencia & \\
\hline Contexto social de las personas & \\
\hline Componente moral en el cuidado & \\
\hline Autocrítica del actuar en enfermería & \\
\hline Mirada de sí mismo & \\
\hline Identificar las desigualdades \\
y sus efectos & \\
\hline Comunicación y capacidad de escucha & \\
\hline Fundamentación del cuidado & \\
\hline Reconocer cambios ante & \\
las experiencias & \\
\hline
\end{tabular}

Fuente: elaboración propia.

2. Análisis de un artículo

Por grupos de máximo tres personas, se asigna un artículo original que es producto de investigación y debe ser escrito por enfermeras; en él el alumno tendrá que identificar:

- Participantes del estudio.

- Contexto donde se desarrolló la investigación.
- Aspectos éticos considerados en la investigación.

- Tema investigado.

- Resultados obtenidos según los objetivos.

- Conclusiones.

Una vez se identifiquen los aspectos anteriores, el grupo analiza la información y la relaciona con los patrones de conocimiento: empírico, ético, sociopolítico, emancipatorio y estético. En este ejercicio el personal no aplicaría.

3. Situación de enfermería

En la narrativa, la enfermera cuenta su experiencia con un lenguaje sencillo, pero a su vez enriquece la descripción de las vivencias y experiencias de cuidado, esto le transmite al alumno los elementos necesarios para identificar los patrones del conocimiento. Una vez se asigne a cada grupo de trabajo una situación de enfermería, se debe revisar e ir haciendo el análisis de los aspectos que son característicos de los patrones del conocimiento. En la tabla 3, se presentan unas preguntas orientadoras para cada patrón de conocimiento.

Tabla 3

Preguntas orientadoras patrones de conocimiento

\begin{tabular}{|c|c|c|}
\hline $\begin{array}{l}\text { Patrones de } \\
\text { conocimiento }\end{array}$ & Preguntas orientadoras & Recomendaciones \\
\hline Empírico & ¿Qué conocimientos requería la enfermera según la situación? & $\begin{array}{l}\text { Dado el caso, indagar términos desconocidos en } \\
\text { diversas fuentes de información. }\end{array}$ \\
\hline Ético & $\begin{array}{l}\text { ¿Qué responsabilidades con el sujeto de cuidado se evidencian } \\
\text { en la situación? } \\
\text { Qué principios se tienen o no en cuenta, según la normatividad? }\end{array}$ & $\begin{array}{l}\text { Revisar los principios expuestos en la Ley } 911 \text { de } \\
2004 \text { y la Ley } 266 \text { de } 1996 . \\
\text { Responsabilidades con el sujeto de cuidado según la } \\
\text { Ley } 911 \text { de } 2004 .\end{array}$ \\
\hline Estético & ¿Cómo describe el proceso comunicativo enfermera- paciente? & \\
\hline Personal & ¿Cómo describe la enfermera en la situación de enfermería? & \\
\hline Emancipatorio & $\begin{array}{l}\text { ¿Cuál es la reflexión que hace la enfermera?? ¿Cómo se sintió al } \\
\text { final de la experiencia? }\end{array}$ & \\
\hline Sociopolítico & $\begin{array}{c}\text { ¿Cómo describe los aspectos sociales o políticos del problema de } \\
\text { la persona? }\end{array}$ & $\begin{array}{l}\text { Revisar el problema identificado y contextualizar } \\
\text { según revisión de fuentes de información adicional. }\end{array}$ \\
\hline
\end{tabular}




\section{REFERENCIAS}

Escobar-Castellanos B, Sanhueza-Alvarado O. Patrones de conocimiento de Carper y expresión en el cuidado de enfermería. Enfermería (Montevideo) [Internet]. 2018 jun. [citado 14 nov. 2020];7(1):27-42. Disponible en: http://dx.doi.org/10.22235/ech.v7i1.1540

Siles González J. La utilidad práctica de la epistemología en la clarificación de la pertinencia teórica y metodológica en la disciplina enfermera. Index Enferm [Internet]. 2016 jun. [citado 19 nov. 2020];25(1-2):86-92. Disponible en: http://scielo.isciii.es/scielo.php?script=sci_arttext\&pid=S1132-12962016000100020\&lng=es.

Jackson JP, Clements PT, Averill JB, Zimbro K. Patterns of knowing: proposing a theory for nursing leadership. Nursing Economics. 2009;27(3):149-159.

Schwind JK, Manankil-Rankin L. Using narrative reflective process to augment personal and aesthetic ways of knowing to support holistic person-centred relational practice. Reflective Practice [Internet]. 2020 (citado 18 nov. 2020);21(4): 473-483. https://doi.org/10.1080/14623943.2020.1777958

Thorne S. Rethinking Carper's personal knowing for 21st century nursing. Nursing Philosophy [Internet]. 2020 (citado 18 nov. 2020);21(4):1-7. https://doi.org/10.1111/nup.12307

Piedrahita Sandoval LE, Rosero Prado AL. Relación entre teoría y praxis en la formación de profesionales de enfermería: revisión integradora de literatura. Enfermería Global [Intenet]. 2017 jul. (citado 17 nov. 2020);16(47):679-706. Disponible en: https://dx.doi.org/10.6018/eglobal.16.3.269261

Vega, P., \& Rivera, M. S. Cuidado holístico, ¿mito o realidad? Horizonte de Enfermería [Internet]. 2009 (citado 18 nov. 2020);20(1:81-86. Disponible en: https://www.imbiomed.com.mx/articulo.php?id=62999

\section{BibLiOgRAFÍA COMPLEMENTARIA}

Escobar-Castellanos B, Jara-Concha, P. Filosofia de Patricia Benner, aplicação em treinamento de enfermagem: propostas de estratégias de aprendizagem. Educación. [Internet]. 2020 Sept 20;28(54):182-202. Disponible en: https://dx.doi.org/10.18800/educacion.201901.009

Cruz Gavilanes MT, Rodríguez Pañora PE, Acero Amay MT, Cruz Gavilanes YN. La motivación de los estudiantes de enfermería. Dominio de las Ciencias. 2017 oct.;3(4):570-587. 


\section{Apartado finAL}

Estructurar la temática y las estrategias de aprendizaje para los alumnos en el desarrollo del curso que fundamenta la epistemología del cuidado y el análisis crítico y reflexivo de los aspectos fundamentales en el marco disciplinar contribuye al fomento y al conocimiento de las teorías propias que le aportan al estudiante para la argumentación de su quehacer y para continuar el desarrollo en su ejercicio profesional. De igual manera, se contribuye a una educación en el ser, hacer y saber para el mundo de la vida, proceso que contrastará al iniciar el proceso de la práctica asistencial. Así, pues, este documento se cierra con la frase de Florence Nightingale: "Nunca nos consideramos enfermeras acabadas ... debemos aprender toda la vida". 


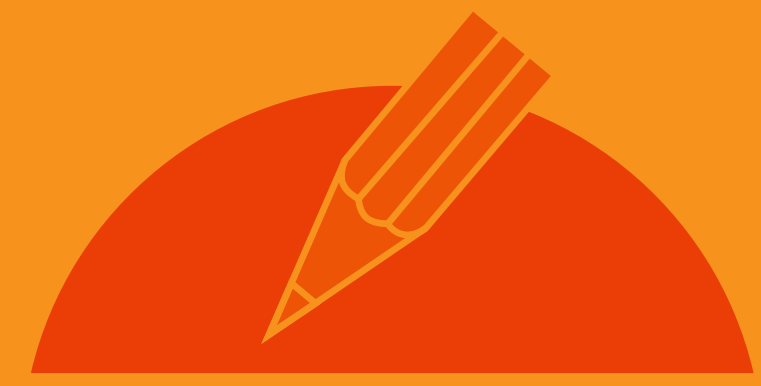

Notas

de clase 